Algol and Related Systems 


\title{
A NEW CATALOGUE OF SEMIDETACHED ALGOL-TYPE BINARIES WITH WELL DETERMINED ABSOLUTE DIMENSIONS
}

\author{
J. M. GARCÍA \\ Departamento de Física, Universidad Politécnica de Madrid \\ Ronda de Valencia, 3. 28012 Madrid, Spain.
}

\begin{abstract}
A. GIMÉNEZ
Laboratorio de Astrofisica Espacial y Física Fundamental (INTA). Apartado 50727. 28080 Madrid, Spain.
\end{abstract}

\begin{abstract}
Data on a selected sample of semidetached Algol-type eclipsing binaries with well-known absolute dimensions have been collected. The strict criteria of selection adopted are given together with the provisional form of the catalogue. This includes besides the absolute dimensions other relevant parameters that may be of interest.
\end{abstract}

\section{Introduction}

In this paper we present a preliminary version of a catalogue of Algol-type binary systems which constitutes part of a systematic study that we are carrying out since some years ago.

This compilation is a first step to set up a homogeneous list of semidetached stars with absolute parameters whose lack was stated on the occasion of the 107 Coll. of the IAU on Algols (Batten, 1989) held in Victoria (Canada) in 1988.

The sample of stars which we present is still limited but we prefer to give greater importance to the quality of the data and to the homogeneity of the whole than to a simple enumeration of objects with small specific information.

Up to now the largest compilation of semidetached systems was published by Budding in 1984: "A Catalogue of Classical (Evolved) Algol Type Binary Candidate Stars" which includes 414 objects. However in such catalogue most of the absolute dimensions are not included and for most of the systems its correspondence with the evolutionary stage of Algols is not known.

In other compilations where absolute parameters are included (Brancewicz and Dworak, 1980; Giuricin and Mardirossian, 1981; Giuricin et al., 1983 ) a high diversity of methods for their evaluation co-exist. The choice of the mass-spectral type relationship for the calculations of the mass of the components is furthermore very frequent.

An analysis of the different applicable methods and their degree of reliability has been made by Giménez and García (1990).

Because of the need to revise the different lists of semidetached systems, our catalogue was elaborated on the basis of severe and uniform criteria for the election of the methods of analysis as well as for the quality of the used spectroscopic and photometric information.

\section{Selection criteria}

1) Only systems with light curves in more than one band have been considered. The lack of measures in several bands makes impossible a check of the values of the geometrical variables 
(i, $\mathrm{r}_{1}, \mathrm{r}_{2}$ ) and of the temperature of the cool component.

2) We have discarded the photometric solutions obtained by means of non-synthetic methods. However not every synthetic method is applicable. Only those using the Roche geometry are valid to analyze stellar configurations of Algol-type. In the existing literature there is a great number of photometric analysis achieved with WINK but this one is only applicable under certain conditions (García, 1990).

3) The effective temperatures of the hot component have been determined from the colour indices. We have used mainly photometric data in the uvby-Hß system of Strömgren-Crawford together with the calibrations of Jackobsen (1985), Moon and Dworetsky (1985) and Davis and Shobbroook (1987). For some stars (S Cnc, U Cep, RS Cep, RY Gem, TT Hya, AW Peg and RW Per ) we have made use of the available spectrophotometric information. The chosen effective temperatures of the cool components is the result of the photometric analyses of the light curves in several colours.

4) The relative radii are obtained by using the following expressions:
a) In WINK $r=\left(\begin{array}{lll}a & b & c\end{array}\right)^{1 / 3}$
b) In WDM $r=\left(r_{\text {back }}+r_{\text {side }}+r_{\text {pole }}\right) \quad$ if $0.18 \leq r \leq 0.55$
$r=\left(r_{\text {back }} r_{\text {side }} r_{\text {pole }}\right)^{1 / 3}$ in other cases

5) The photometrically obtained mass ratio was only considered to check results, because the light curves of the classic Algol-type systems do not contain enough information to obtain $q$ by means of least squares fitting.

6) Special care was taken about the source of the values $K_{1}$ and $K_{2}$ (semiamplitude of the radial velocity curves) because even in those cases in which the velocity variations of both components are detected, there are several effects which may mask the real orbital movement (Popper, 1989). The selection of the adequate lines for the measurement of the radial velocities is one of the more important aspects in order to obtain semiamplitudes free from systematic errors.

7) Masses deduced from the spectral type have been not taken into account. Frequently the real photospheric spectrum is unknown and the use of a mean mass-spectral type relation, valid for the main sequence, bears a high degree of inaccuracy (Nordström, 1989).

8) When $K_{2}$ is unknown the mass function, $f(m)$, is used together with the mass ratio $q_{E D}$, obtained through the method described by Giménez y García (1990).

\section{Description of the catalogue}

The 43 semidetached systems selected according to the above mentioned criteria will be published separately in a catalogue which contents are arranged in columns as follows:

column 1: star name

2: magnitude

3: spectral type

4: period in days

5: mass ratio obtained spectroscopically if the star is double-lined ( $K_{1}$ and $K_{2}$ known). In the other cases it corresponds to the value of $\mathrm{q}_{\mathrm{ED}}$.

6: orbital inclination in degrees

7 and 8: relative radii of the components obtained photometrically

9 and 10: semiamplitudes of the radial velocity curves in $\mathrm{km} / \mathrm{s}$

11 and 12: masses of the components in solar units 
column 13 and 14: radii of the components in solar units

15 and 16: logarithm of the effective temperature of both components

17: main references

Figures 1 and 2 show the distribution of $\log P$ and that of the mass ratio respectively, for the systems included in the catalogue. In the $q-\log P$ plane, the known effect that lower mass ratios tend to go with longer orbital periods is present. As it has been pointed out by some authors this fact is related with mass transfer processes occurred in a close binary. We have studied more in deep the scenario of mass transfer using the observational data of the semidetached binaries considered in our catalogue (García and Giménez, 1991).

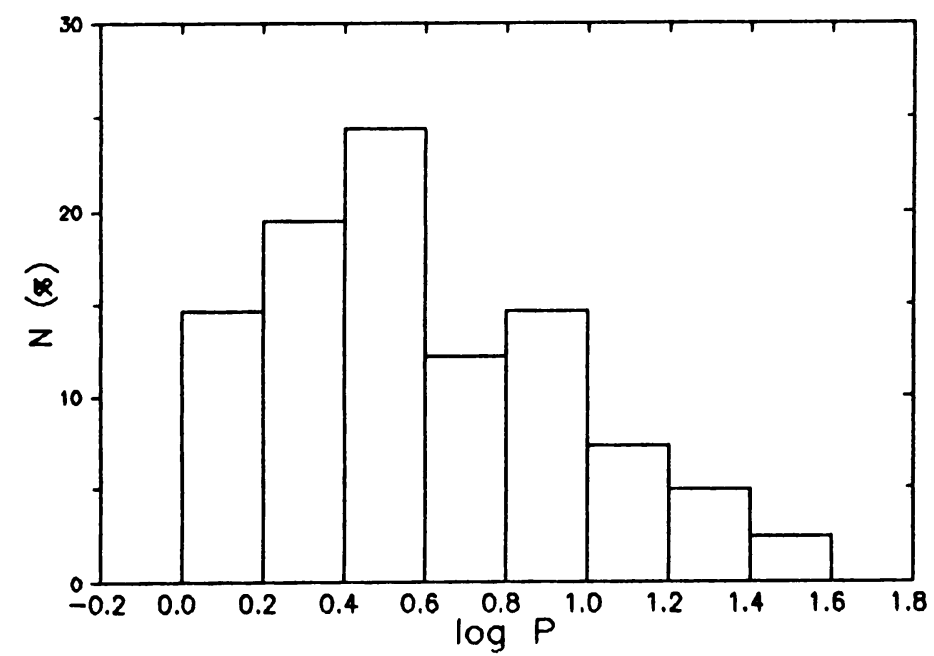

Figure 1. Percentage of Algols falling into consecutive intervals of 0.2 in the logarithm of the period.

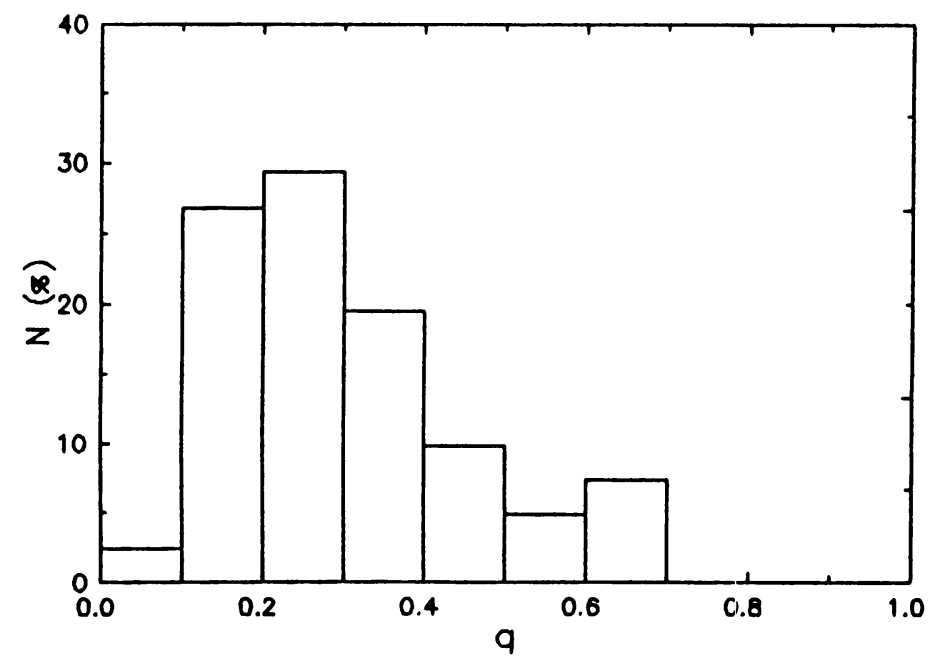

Figure 2. Mass ratio distribution for the 43 semidetached Algol-type systems considered in our catalogue 


\section{Acknowledgements}

This work has been supported by the Spanish DGCYT through project PB87-0235

\section{References}

Batten, A. (1989), Algols, ed. A.H. Batten, Kluwer Academic Publishers, Dordrecht.

Brancewicz, H.K. and Dworak, T.Z. (1980), Acta Astron. 30, 501

Davis, J. and Shobbrook, R.R. (1977), Mont. Not. Roy. Astron. Soc. 178, 651

García, J.M. (1990), Doctoral Thesis. Univ. Complutense de Madrid.

García, J.M. and Giménez, A. (1991) "Mass Transfer Characterization in Close Binary Stars", Communication to this Symposium.

Giménez, A. and García, J.M. (1990), in Active Close Binaries, ed. C.Ibanoglu, NATO ASI, p.121 Giuricin, G. and Mardirossian, F. (1981), Astrophys. J. Suppl. 46, 1

Giuricin, G., Mardirosian, F. and Mezzetti, M. (1983), Astrophys. J. Suppl. 52, 35. Jakobsen, A.M. (1985), Preprint, private comunication.

Moon, T. T. and Dworetsky, M.M. (1985), Mont. Not. Roy. Astron. Soc. 217, 305

Nordström, B. (1989), Astrophys. J. 341, 934.

Popper, D.M. (1989), Astrophys. J. 71, 595. 